\title{
The C-reactive protein/albumin ratio predicts overall survival of patients with advanced pancreatic cancer
}

\author{
Mengwan Wu ${ }^{1} \cdot$ Jing Guo $^{1} \cdot$ Lihong Guo $^{1} \cdot$ Qiang Zuo $^{1}$
}

Received: 12 February 2016 / Accepted: 13 June 2016/Published online: 25 June 2016

(C) The Author(s) 2016. This article is published with open access at Springerlink.com

\begin{abstract}
Recent studies have demonstrated the prognostic value of the C-reactive protein/albumin (CRP/Alb) ratio in cancer. However, the role of the CRP/Alb ratio in advanced pancreatic cancer (PC) has not been examined. A retrospective study of 233 patients with advanced $\mathrm{PC}$ was conducted. We investigated the relationship between the $\mathrm{CRP} / \mathrm{Alb}$ ratio, clinicopathological variables, and overall survival (OS). The optimal cutoff point of the $\mathrm{CRP} / \mathrm{Alb}$ ratio was 0.54 . A higher $\mathrm{CRP} / \mathrm{Alb}$ ratio was significantly associated with an elevated neutrophil-lymphocyte ratio (NLR) $(P<0.001)$ and higher modified Glasgow prognostic score (mGPS) $(P<0.001)$. Using univariate analyses, we found that the age $(P=0.009)$, disease stage $(P<0.001)$, NLR $(P<0.001)$, mGPS $(P<0.001)$, and CRP/Alb ratio $(P<0.001)$ were significant predictors of OS. Patients with a higher CRP/Alb ratio had a worse OS than patients with a lower CRP/Alb ratio (hazard ratio (HR) 3.619; $95 \%$ CI 2.681-4.886; $P<0.001$ ). However, the CRP/Alb ratio was identified as the only inflammation-based parameter with an independent prognostic ability in the multivariate analyses $(P<0.001)$. The pretreatment $\mathrm{CRP} / \mathrm{Alb}$ ratio is a superior prognostic and therapeutic predictor of OS in advanced PC.
\end{abstract}

Keywords Advanced pancreatic cancer · Inflammation . $\mathrm{CRP} /$ Alb ratio $\cdot$ Prognostic score $\cdot$ Survival

Qiang Zuo

nfyyzq@126.com

1 Department of Oncology, Nanfang Hospital, Southern Medical University, 1838 North Guangzhou Avenue, Guangzhou, Guangdong Province 510515, China

\section{Introduction}

Pancreatic cancer ranks as the 12th most commonly diagnosed cancer and seventh leading cause of cancer death; there are an estimated 337,900 new cases and 330,400 deaths occurring in 2012 worldwide [1]. Most patients are diagnosed at an advanced stage and have a rapid clinical decline, culminating in death within less than 1 year after diagnosis. The high mortality is associated with a limited chance of curative resection at the time of diagnosis, as surgical resection offers the only prospect of long-term survival or cure. Moreover, one study showed that the median survival in patients with curative resection was 14.9 months longer than in those with an advanced stage (19.1 vs 4.2 months). Similarly, the 5-year survival rates were higher in the operable patients (19.3 vs $0.8 \%$ ) [2]. Although surgical resection has improved the prognosis of pancreatic cancer patients, it continues to have a poor prognosis.

Currently, although continuous advances in modern diagnostic imaging have been achieved, most established prognostic factors continue to rely on surgical exploration, such as the tumor size, histologic grade, and vascular and nodal involvement. Many patients at an advanced stage also undergo a morbid operative procedure during the course of pancreatic lesion evaluation. Therefore, it is important to identify some easily obtainable and reliable prognostic factors for better risk stratification and optimal treatment plans. With the increasing number of studies suggesting that tumor-elicited inflammation plays a key role in malignant transform and tumor progression [3-6], some inflammation-based prognostic factors have been explored in many cancers during the course from bench to bedside. These factors are all easily available from peripheral blood samples, including the neutrophillymphocyte ratio (NLR), platelet-lymphocyte ratio 
(PLR), white cell count and C-reactive protein (CRP) combined into the prognostic index (PI), albumin and lymphocyte count in the prognostic nutritional index (PNI), and CRP- and albumin-based factors of the modified Glasgow prognostic score (mGPS) and C-reactive protein/albumin (CRP/Alb) ratio [7-12]. Among these indicators, the CRP/Alb ratio has been reported as a novel reliable marker in different cancers such as lung, liver, gastric, and esophageal cancer [8, 13-16]. However, the role of the CRP/Alb ratio in patients with advanced pancreatic cancer has not previously been elucidated.

In this retrospective study, we examined the prognostic value of the CRP/Alb ratio and compared it to other established inflammation-based prognostic scores.

\section{Materials and methods}

\section{Patients}

We enrolled 233 patients who were diagnosed with advanced pancreatic cancer in Nanfang Hospital of Southern Medical University (Guangzhou, Guangdong Province, China) between January 2011 and December 2014. All medical records were retrospectively reviewed. The following inclusion criteria were used: (1) cytologically or histologically diagnosed as pancreatic adenocarcinoma; (2) survival of at least 30 days after diagnosis; (3) confirmed stage III or IV based on the AJCC/UICC TNM staging system (the 7th edition); and (4) pretreatment laboratory data were available. Patients suffering from detectable acute inflammation were excluded. Selected patients were carefully followed up after pathological diagnosis until September 30, 2015, or death from any cause.

\section{Clinical data collection}

Baseline characteristics were obtained from the electronic medical record system, including the age; gender; tumor location and stage; pretreatment laboratory counts of white cells, neutrophils, lymphocytes, and platelets; tumor markers (carcinoembryonic antigen 19-9, CA19-9, and carcinoembryonic antigen (CEA)); levels of CRP and albumin; and therapeutic information. Based on previous studies, the CRP/Alb ratio was calculated by dividing the serum CRP level by the serum albumin level [17]. The mGPS combined the CRP and albumin concentrations. Patients who had both elevated CRP $(>1 \mathrm{mg} / \mathrm{dl})$ and decreased albumin levels $(<3.5 \mathrm{~g} / \mathrm{dl})$ were assigned a score of 2 . Patients with only elevated CRP $(>1 \mathrm{mg} / \mathrm{dl})$ were assigned a score of 1 , and patients with neither of these abnormalities were assigned a score of 0 [18]. NLR and PLR were both
Table 1 Clinicopathological characteristics of patients with pancreatic cancer $(n=233)$

\begin{tabular}{|c|c|}
\hline Factor & Number (\%) \\
\hline \multicolumn{2}{|l|}{ Age (years) } \\
\hline$<62$ & $111(47.6)$ \\
\hline$\geq 62$ & $122(52.4)$ \\
\hline \multicolumn{2}{|l|}{ Gender } \\
\hline Female & $77(33.0)$ \\
\hline Male & $156(67.0)$ \\
\hline \multicolumn{2}{|l|}{ Disease stage } \\
\hline Locally advanced & $83(35.6)$ \\
\hline Metastasis & $150(64.4)$ \\
\hline \multicolumn{2}{|l|}{ Location } \\
\hline Head & $104(44.6)$ \\
\hline Body & $64(27.5)$ \\
\hline Tail & 37 (15.9) \\
\hline Diffusion & $28(12.0)$ \\
\hline \multicolumn{2}{|l|}{$\mathrm{CRP} / \mathrm{Alb}$} \\
\hline$<0.54$ & $159(68.2)$ \\
\hline$\geq 0.54$ & $74(31.8)$ \\
\hline \multicolumn{2}{|l|}{ NLR } \\
\hline$<5$ & $176(75.5)$ \\
\hline$\geq 5$ & $57(24.5)$ \\
\hline \multicolumn{2}{|l|}{ PLR } \\
\hline$<150$ & $115(49.4)$ \\
\hline$\geq 150$ & $118(50.6)$ \\
\hline \multicolumn{2}{|l|}{ mGPS } \\
\hline 0 & $119(51.1)$ \\
\hline 1 & $76(32.6)$ \\
\hline 2 & $38(16.3)$ \\
\hline \multicolumn{2}{|l|}{ CA19-9 } \\
\hline$<\operatorname{Median}(427 \mathrm{U} / \mathrm{ml})$ & $116(49.4)$ \\
\hline$>$ Median & $117(50.6)$ \\
\hline \multicolumn{2}{|l|}{ CEA } \\
\hline$<\operatorname{Median}(3.46 \mathrm{ng} / \mathrm{ml})$ & $116(49.4)$ \\
\hline$>$ Median & $117(50.6)$ \\
\hline \multicolumn{2}{|l|}{ Treatment } \\
\hline No chemotherapy & $71(30.5)$ \\
\hline Chemotherapy & $162(69.5)$ \\
\hline \multicolumn{2}{|l|}{ End-point } \\
\hline Alive & $6(2.6)$ \\
\hline Dead & $227(97.4)$ \\
\hline
\end{tabular}

$C R P / A l b$ C-reactive protein/albumin, $N L R$ neutrophil-lymphocyte ratio, $P L R$ platelet/lymphocyte ratio, $m G P S$ the modified Glasgow prognostic score, CA19-9 carbohydrate antigen 19-9, CEA carcinoembryonic antigen

categorized into two groups according to the cutoff values of $>5$ and $>150$, respectively $[19,20]$. 


\section{Statistical analyses}

Comparisons between groups were performed using the chisquare test. The optimal cutoff value of the CRP/Alb ratio was determined using a web-based system, R softwareengineered, which was designed by Budczies $\mathrm{J}$ et al. (http://molpath.charite.de/cutoff/) [21]. The overall survival (OS) was measured from the day of pathological diagnosis to death from any cause or the final date of follow-up. The OS curves were generated using the Kaplan-Meier method, and differences between groups were compared using the logrank test. Prognostic variables that were significant in univariate analyses were selected for multivariate Cox proportional hazard model analyses using the forward stepwise method. Statistical analyses were performed with SPSS 21.0 (IBM Corporation, Armonk, NY, USA). A two-sided $P$ value $<0.05$ was considered statistically significant.

\section{Results}

\section{Patient characteristics}

A total of 233 patients who were diagnosed with advanced pancreatic cancer in Nanfang Hospital were evaluated. They had a median age of 62 years (range 26-85). Among these patients, $156(67.0 \%)$ were males and $77(33.0 \%)$ were females, while the numbers of patients with locally advanced and metastasis disease were $83(35.6 \%)$ and 150 $(64.4 \%)$, respectively. Nearly half of patients had a primary pancreatic head tumor $(n=104,44.6 \%)$. The pretreatment blood sample analyses showed that the median CA19-9 lev- el for the entire group was $427 \mathrm{U} / \mathrm{ml}$, while the CEA was a median of $3.45 \mathrm{ng} / \mathrm{ml}$. More than two thirds of patients received gemcitabine monotherapy as first-line treatment $(n=162,69.5 \%)$. The baseline characteristics of the 233 patients are shown in Table 1.

\section{Cutoff point determination for the CRP/Alb ratio}

The median value of the CRP/Alb ratio was 0.241 (range, $0.002-6.728$ ). The analyses that were performed with the biostatistical tool Cutoff Finder showed that a wide range of cutoff points for the CRP/Alb ratio were significant (Fig. 1). For overall survival, the optimal cutoff point of the CRP/Alb ratio for stratifying patients with advanced pancreatic cancer (PC) was 0.54. Based on this result, all patients were categorized into the $\mathrm{CRP} / \mathrm{Alb}-\mathrm{high}(n=74,31.8 \%)$ and CRP/Alblow $(n=159,68.2 \%)$ groups.

\section{Correlation of the CRP/Alb ratio with clinicopathological parameters}

The relationships between the CRP/Alb ratio and other variables are shown in Table 2. A higher CRP/Alb ratio was significantly associated with an elevated NLR $(P<0.001)$ and higher mGPS score $(P<0.001)$. In addition, fewer patients in the high $\mathrm{CRP} / \mathrm{Alb}$ ratio group received chemotherapy $(P<0.001)$ compared to the low $\mathrm{CRP} / \mathrm{Alb}$ ratio group. However, there were no significant differences in the age $(P=0.943)$, gender $(P=0.103)$, disease stage $(P=0.200)$, tumor location $(P=0.084)$, PLR values $(P=0.242)$, CA19-9 levels $(P=0.100)$, and CEA levels $(P=0.100)$ between the $\mathrm{CRP} /$ Alb-high and CRP/Alb-low groups.
Fig. 1 The hazard ratio (HR) for progression-free survival according to the cutoff points of the CRP/Alb ratio in patients with advanced PC. A vertical line designates the chosen cutoff point. The plots were generated using Cutoff Finder

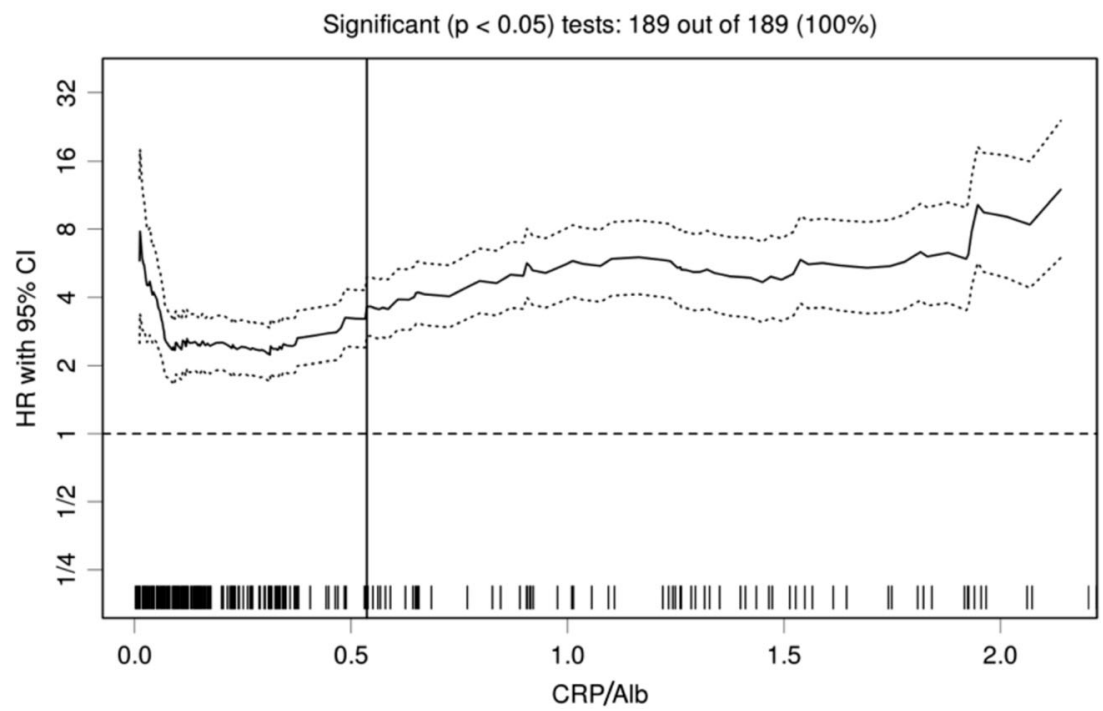


Table 2 Correlation of the CRP/Alb ratio and clinicopathological characteristics of $\mathrm{PC}$ patients

\begin{tabular}{|c|c|c|c|}
\hline Characteristics & $\begin{array}{l}\text { CRP/Alb ratio } \\
<0.54, n(\%)\end{array}$ & $\begin{array}{l}\text { CRP/Alb ratio } \\
\geq 0.54 n(\%)\end{array}$ & $P$ value \\
\hline \multicolumn{3}{|l|}{ Age } & \multirow[t]{3}{*}{0.943} \\
\hline$<62$ & $76(47.8)$ & $35(47.3)$ & \\
\hline$\geq 62$ & $83(52.2)$ & $39(52.7)$ & \\
\hline \multicolumn{3}{|l|}{ Gender } & \multirow[t]{3}{*}{0.103} \\
\hline Female & $58(36.5)$ & $19(25.7)$ & \\
\hline Male & $101(63.5)$ & $55(74.3)$ & \\
\hline \multicolumn{3}{|l|}{ Disease stage } & \multirow[t]{3}{*}{0.200} \\
\hline Locally advanced & $61(38.4)$ & $22(29.7)$ & \\
\hline Metastasis & $98(61.6)$ & $52(70.3)$ & \\
\hline \multicolumn{3}{|l|}{ Location } & \multirow[t]{5}{*}{0.084} \\
\hline Head & $67(42.1)$ & $37(50.0)$ & \\
\hline Body & $43(27.1)$ & $21(28.4)$ & \\
\hline Tail & $24(15.1)$ & $13(17.6)$ & \\
\hline Diffusion & $25(15.7)$ & $3(4.0)$ & \\
\hline \multicolumn{3}{|l|}{ NLR } & \multirow[t]{3}{*}{$0.000 *$} \\
\hline$<5$ & $137(86.2)$ & $39(52.7)$ & \\
\hline$\geq 5$ & $22(13.8)$ & $35(47.3)$ & \\
\hline \multicolumn{3}{|l|}{ PLR } & \multirow[t]{3}{*}{0.242} \\
\hline$<150$ & $84(52.8)$ & $33(44.6)$ & \\
\hline$\geq 150$ & $75(47.2)$ & $41(55.4)$ & \\
\hline \multicolumn{3}{|l|}{ mGPS } & \multirow[t]{4}{*}{$0.000^{*}$} \\
\hline 0 & $119(74.8)$ & $0(0)$ & \\
\hline 1 & $34(21.4)$ & $42(56.8)$ & \\
\hline 2 & $6(3.8)$ & $32(43.2)$ & \\
\hline \multicolumn{3}{|l|}{ CA19-9 } & \multirow[t]{3}{*}{0.100} \\
\hline$<\operatorname{Median}(427 \mathrm{U} / \mathrm{ml})$ & $85(53.5)$ & $31(41.9)$ & \\
\hline$>$ Median & $74(46.5)$ & $43(58.1)$ & \\
\hline \multicolumn{3}{|l|}{ CEA } & \multirow[t]{3}{*}{0.100} \\
\hline$<\operatorname{Median}(3.46 \mathrm{ng} / \mathrm{ml})$ & $85(53.5)$ & $31(41.9)$ & \\
\hline$>$ Median & $74(46.5)$ & $43(58.1)$ & \\
\hline \multicolumn{3}{|l|}{ Treatment } & \multirow[t]{3}{*}{$0.049 *$} \\
\hline No chemotherapy & $42(26.4)$ & $29(39.2)$ & \\
\hline Chemotherapy & $117(73.6)$ & $45(60.8)$ & \\
\hline
\end{tabular}

* Significant differences between patients with the CRP/Alb $<0.54$ and patients with the CRP/Alb $\geq 0.54$

\section{Association between the CRP/Alb ratio and OS}

The results of the univariate and multivariate analyses are presented in Table 3. The median survival time of all of the patients was 4.4 months (95 \% CI 4.16-4.58 months), and 227 (97.4\%) patients died by their final follow-up visit. Based on the cutoff point of the $\mathrm{CRP} / \mathrm{Alb}$ ratio, patients were divided into two groups $(<0.54, n=159$ and $\geq 0.54, n=74)$. In the univariate analyses of survival, the low $\mathrm{CRP} / \mathrm{Alb}$ ratio group had a longer median overall survival than the high $\mathrm{CRP} / \mathrm{Alb}$ ratio group (5.0 vs 2.9 months, $P<0.001)$. Additionally, when patients were stratified by disease stage, the mortality rate of patients with increased C-reactive protein/albumin ratio (CAR) was also higher in both the locally advanced subgroup (5.9 vs 4.1 months, $P<0.001)$ and metastasis subgroup (4.5 vs 2.4 months, $P<0.001$ ) (Fig. 2).

Univariate analyses also showed that older age ( $\geq 62$ vs $<62$ years, $P=0.009$ ), metastasis stage (metastasis vs locally advanced, $P<0.001$ ), an elevated NLR ( $\geq 5$ vs $<5, P<0.001)$, higher mGPS scores $(1+2$ vs $0, P<0.001)$, and nonchemotherapeutic treatment (no chemotherapy vs chemotherapy, $P<0.001$ ) were significantly associated with worse OS in advanced $\mathrm{PC}$.

To investigate whether $\mathrm{CRP} / \mathrm{Alb}$ ratio could serve as an independent prognostic factor in advanced $\mathrm{PC}$, multivariate analyses were also conducted. In the multivariate analyses, patients in the higher CRP/Alb group had a worse outcome than those in the lower ratio group (hazard ratio (HR) 3.995; $95 \%$ CI 2.644-6.034; $P<0.001$ ) (Table 3). In addition, the age $(P<0.001)$, disease stage $(P<0.001)$, and chemotherapy $(P<0.001)$ independently and significantly predicted OS, whereas the NLR, PLR, and mGPS did not predict OS (Table 3 and Fig. 3). Moreover, subgroup analyses were also conducted. Although patients in the CRP/Alb-high group had a worse OS than the CRP/Alb-low group, chemotherapy still contributed to a significantly longer median survival in both groups (CRP/Alb $<0.54$ group, 5.7 vs 3.0 months, $P<0.001$; $\mathrm{CRP} / \mathrm{Alb} \geq 0.54$ group, 3.7 vs 2.0 months, $P<0.001$ ) (Fig. 3).

To identify the interaction between the $\mathrm{CRP} / \mathrm{Alb}$ ratio and mGPS for OS, we categorized patients into four groups based on two indexes, including mGPS score of 1 and CRP/Alb ratio $<0.54$; mGPS score of 1 and $\mathrm{CRP} / \mathrm{Alb}$ ratio $\geq 0.54$; mGPS score of 2 and $\mathrm{CRP} / \mathrm{Alb}$ ratio $<0.54$; and mGPS score of 2 and $C R P / A l b$ ratio $\geq 0.54$. The survival analyses indicated that compared with patients who had an mGPS score of 1 and $\mathrm{CRP} / \mathrm{Alb}$ ratio $\geq 0.54$, those with an mGPS score of 1 and $\mathrm{CRP} / \mathrm{Alb}$ ratio $<0.54$ had a longer median OS (4.5 vs 3.2 months, $P<0.001$ ), which was similar to the comparison of an mGPS score of 2 and CRP/Alb ratio $<0.54$ with an mGPS score of 2 and CRP/Alb ratio $\geq 0.54$ (4.4 vs 2.4 months, $P=0.023)$. However, no significant differences were found between an mGPS score of 1 and CRP/Alb ratio $<0.54$ and an mGPS score of 2 and CRP/Alb ratio $<0.54$ (4.5 vs 4.4 months, $P=0.370$ ) or between an mGPS score of 1 and $\mathrm{CRP} / \mathrm{Alb}$ ratio $\geq 0.54$ and an $\mathrm{mGPS}$ score of 2 and $\mathrm{CRP} / \mathrm{Alb}$ ratio $\geq 0.54$ (3.2 vs 2.4 months, $P=0.416$ ) (Fig. 4).

\section{Discussion}

Despite recent improvements in the validated benefit of chemotherapy and spread of multidisciplinary therapy for patients in the advanced stage, pancreatic cancer remains a devastating disease with an extremely poor prognosis. Optimal responses will be achieved only if the treatment plan is tailored for individuals based on accurate and stable prediction of potential survival. However, tumor-related parameters such as the pathological 
Table 3 Prognostic factors for overall survival identified by univariate and multivariate analyses

\begin{tabular}{|c|c|c|c|c|}
\hline & \multicolumn{2}{|l|}{ Univariate analysis } & \multicolumn{2}{|l|}{ Multivariate analysis } \\
\hline & HR (95 \% CI) & $P$ value & HR $(95 \%$ CI $)$ & $P$ value \\
\hline \multicolumn{5}{|l|}{ Age (years) } \\
\hline$<62$ & Ref & & Ref & \\
\hline$\geq 62$ & $1.419(1.089-1.850)$ & $0.009 *$ & $1.681(1.280-2.209)$ & $0.000^{*}$ \\
\hline \multicolumn{5}{|l|}{ Gender } \\
\hline Female & Ref & & & \\
\hline Male & $1.099(0.834-1.449)$ & 0.501 & & \\
\hline \multicolumn{5}{|l|}{ Disease stage } \\
\hline Locally advanced & Ref & & Ref & \\
\hline Metastasis & $2.317(1.745-3.076)$ & $0.000 *$ & $2.620(1.939-3.540)$ & $0.000 *$ \\
\hline \multicolumn{5}{|l|}{ Location } \\
\hline Head & Ref & & & \\
\hline Body & $0.846(0.553-1.295)$ & 0.441 & & \\
\hline Tail & $0.894(0.567-1.408)$ & 0.627 & & \\
\hline Diffusion & $0.962(0.582-1.590)$ & 0.880 & & \\
\hline \multicolumn{5}{|l|}{ CRP/Alb } \\
\hline$<0.54$ & Ref & & Ref & \\
\hline$\geq 0.54$ & $3.619(2.681-4.886)$ & $0.000 *$ & $3.995(2.644-6.034)$ & $0.000^{*}$ \\
\hline \multicolumn{5}{|l|}{ NLR } \\
\hline$<5$ & Ref & & Ref & \\
\hline$\geq 5$ & $2.564(1.870-3.517)$ & $0.000 *$ & $1.049(0.734-1.499)$ & 0.795 \\
\hline \multicolumn{5}{|l|}{ PLR } \\
\hline$<150$ & Ref & & & \\
\hline$\geq 150$ & $1.260(0.969-1.639)$ & 0.085 & & \\
\hline \multicolumn{5}{|l|}{ mGPS } \\
\hline 0 & Ref & & Ref & \\
\hline $1+2$ & $2.369(1.806-3.109)$ & $0.000 *$ & $1.226(0.829-1.812)$ & 0.308 \\
\hline \multicolumn{5}{|l|}{ CA19-9 } \\
\hline$<\operatorname{Median}(427 \mathrm{U} / \mathrm{ml})$ & Ref & & & \\
\hline$>$ Median & $1.104(0.849-1.436)$ & 0.459 & & \\
\hline \multicolumn{5}{|l|}{ CEA } \\
\hline$<\operatorname{Median}(3.46 \mathrm{ng} / \mathrm{ml})$ & Ref & & & \\
\hline$>$ Median & $1.298(0.998-1.688)$ & 0.052 & & \\
\hline \multicolumn{5}{|l|}{ Treatment } \\
\hline No chemotherapy & Ref & & Ref & \\
\hline Chemotherapy & $0.146(0.103-0.205)$ & $0.000^{*}$ & $0.174(0.121-0.250)$ & $0.000 *$ \\
\hline
\end{tabular}

* Significant differences between patients with the CRP/Alb $<0.54$ and patients with the $\mathrm{CRP} / \mathrm{Alb} \geq 0.54$

stage and resectability of the primary tumor primarily play a role in evaluating disease and predicting the outcome.

Therefore, an increasing number of thorough studies have identified some easily available predictors for substituting the traditional surgical method. A diverse set of observations offers evidence supporting the correlation between inflammation and cancer. While early studies were devoted to elucidating the link between pre-existing inflammation and consequent tumor initiation, more recent studies tend to add new insight into tumor-associated inflammation and the host response to oncogenic change in the intrinsic pathway. For the intrinsic pathway, inflammation and tumor growth are driven by genetic mutations that cause the activation of transcription factors, release of inflammatory mediators (chemokines, cytokines, and prostaglandins), and subsequent infiltration of inflammatory cells and angiogenesis [5]. This inflammatory condition further promotes the malignant progression of tumors, enhances local immunosuppression, induces invasion and metastatic spread, and influences the treatment response [5, 22, 23]. 
A

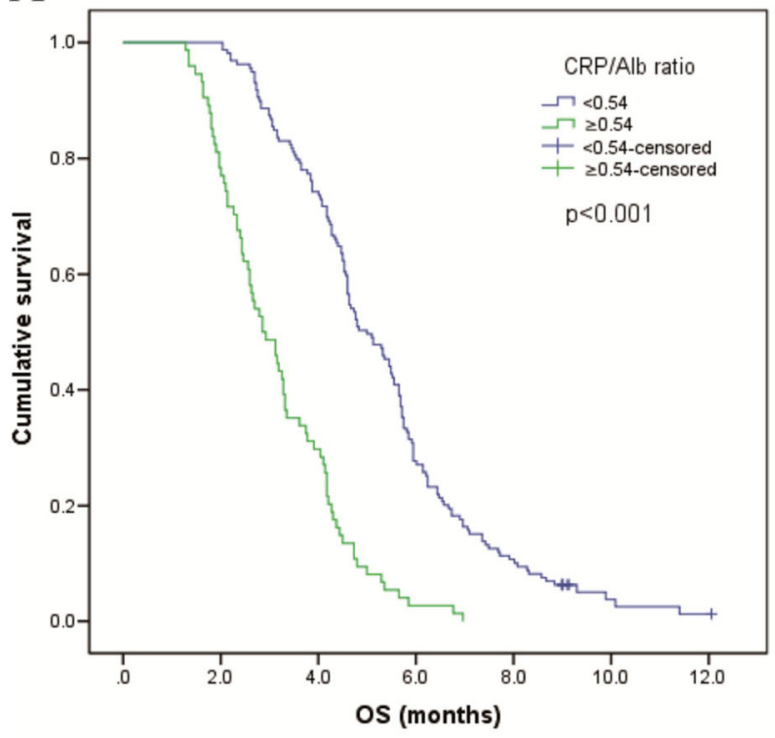

B

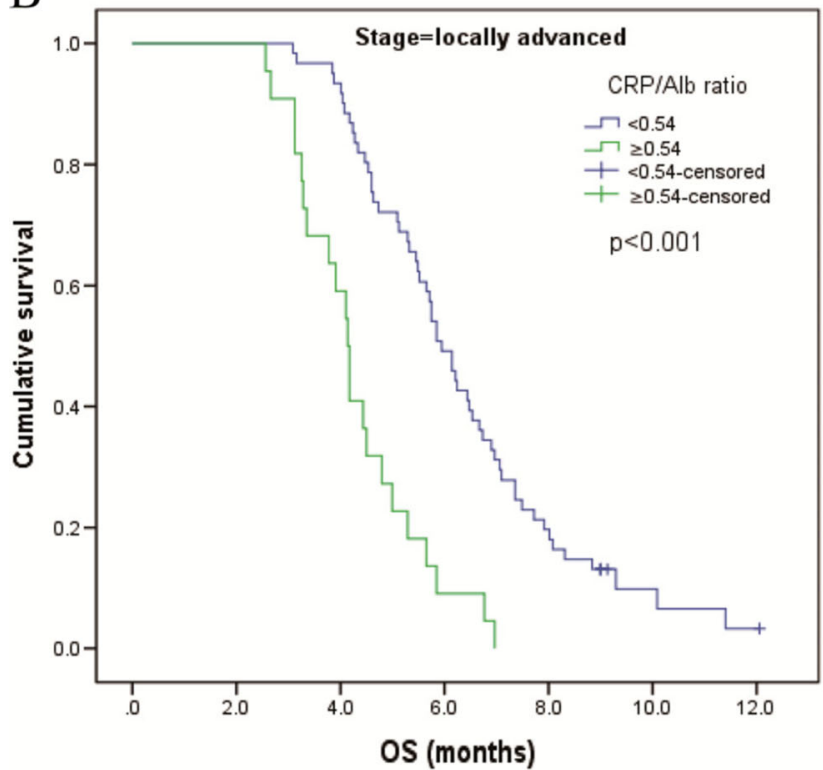

C

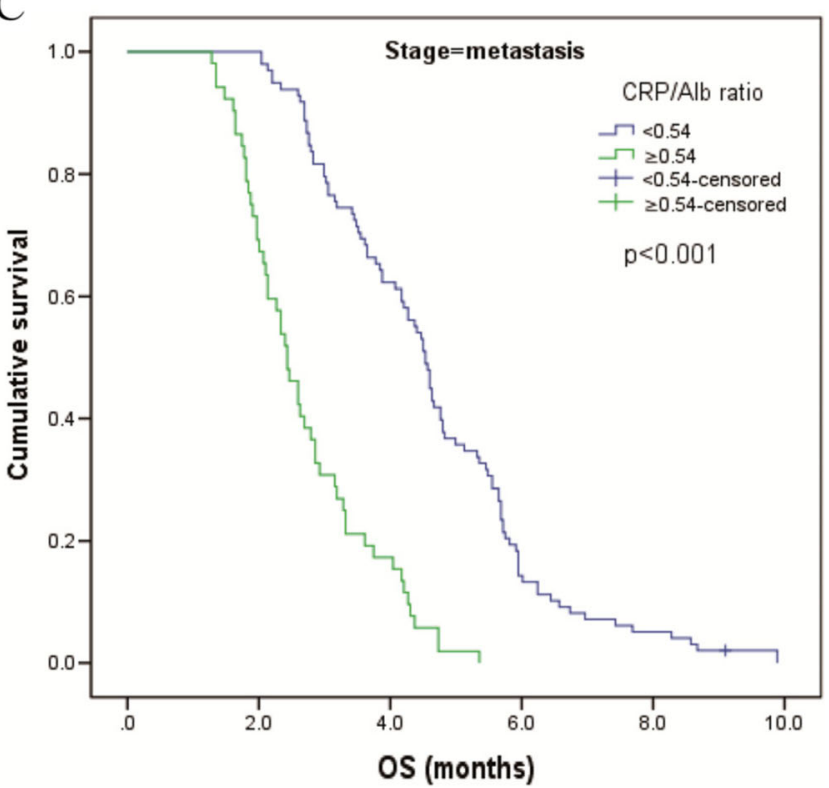

Fig. 2 Kaplan-Meier survival curves showing the difference between the high CRP/Alb ratio and low CRP/Alb ratio groups a in all patients, $\mathbf{b}$ in patients with locally advanced disease, and $\mathbf{c}$ in patients with metastatic disease

Among the many inflammatory mediators, interleukin-6 (IL-6) is a notable inflammatory cytokine secreted by innate immune cells that regulates the levels of C-reactive protein (CRP), an acute-phase protein [24]. Recently, it was reported that elevated pretreatment serum IL-6 and CRP levels showed a significant relationship with a poor outcome [25-28]. Additionally, the serum albumin level is not only a reflection of nutritional state but also it is, to a larger degree, a consequence of the inflammatory state. Plausible explanations are as follows. When considered with tumor-related systematic inflammation, the ability of the liver to produce albumin decreases as a result of increased acute-phase protein synthesis
$[29,30]$. Alternatively, the release of cytokines from inflammatory cells increases the microvascular permeability, increasing the flow of serum albumin towards the extravascular compartment [31]. Therefore, the link between hypoalbuminemia and inflammation is also quite strong [32]. Meanwhile, the role of the pretreatment serum albumin levels as an independent predictor of the OS has been demonstrated in various cancers, including PC [33-35].

These achievements greatly piqued our interest in combining CRP and albumin into a noble potential inflammatory prognostic indicator in advanced PC and identifying its predictive value. In addition, some recent studies have 

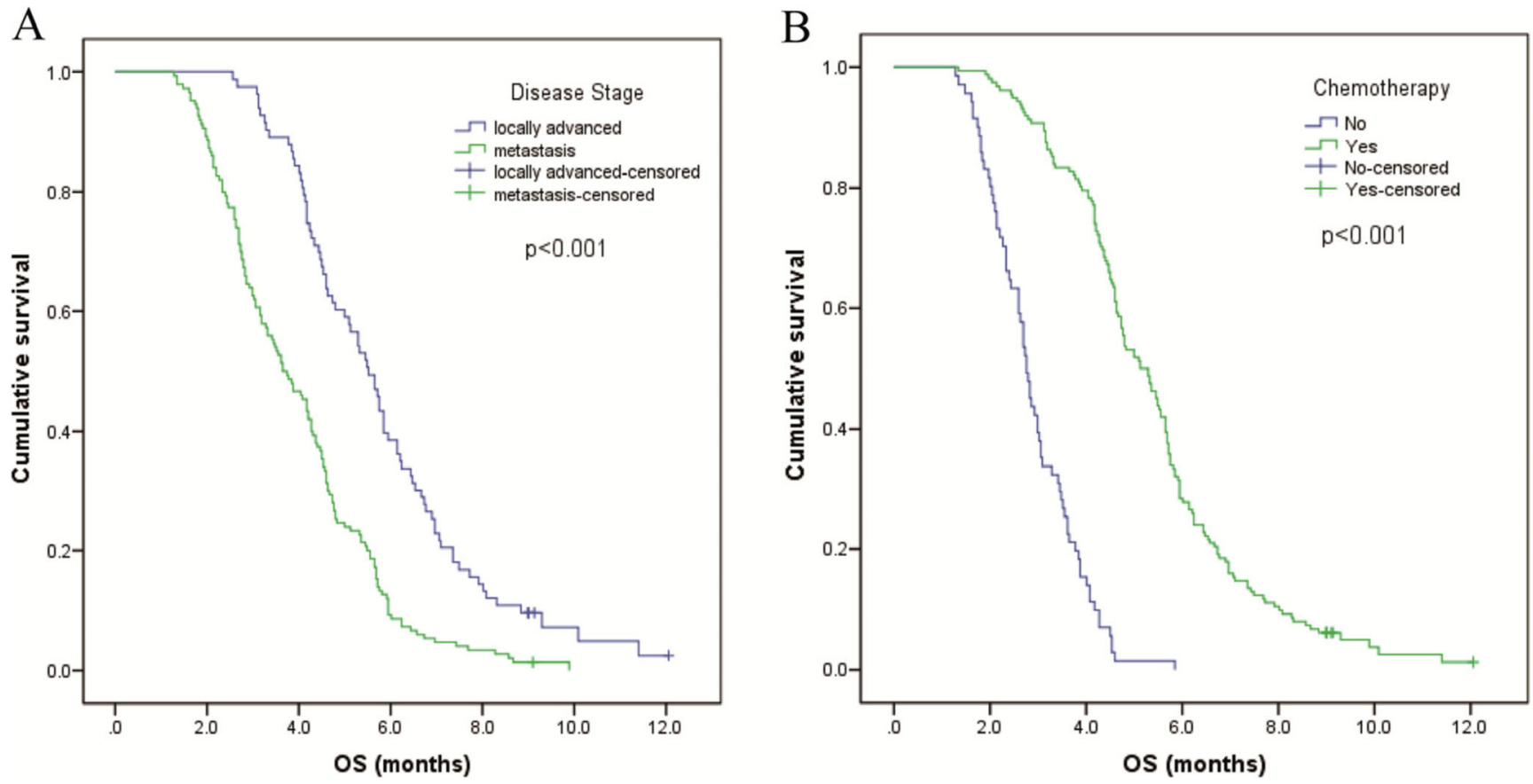

Fig. 3 The overall survival curves according to $\mathbf{a}$ disease stage, and $\mathbf{b}$ treatment with or without chemotherapy

demonstrated that the $\mathrm{CRP} / \mathrm{Alb}$ ratio is a promising inflammation-associated prognostic factor in cancer, including liver, lung, gastric, and esophageal cancer [8, 13-17]. In our study, we assessed and compared the prognostic value of the NLR, PLR, mGPS, and CRP/Alb ratio in advanced PC by retrospectively analyzing the pretreatment laboratory data of 233 eligible patients. According to statistical analysis, there was a significant association between the CRP/Alb ratio and other inflammatory indexes (excluding PLR), which might suggest that

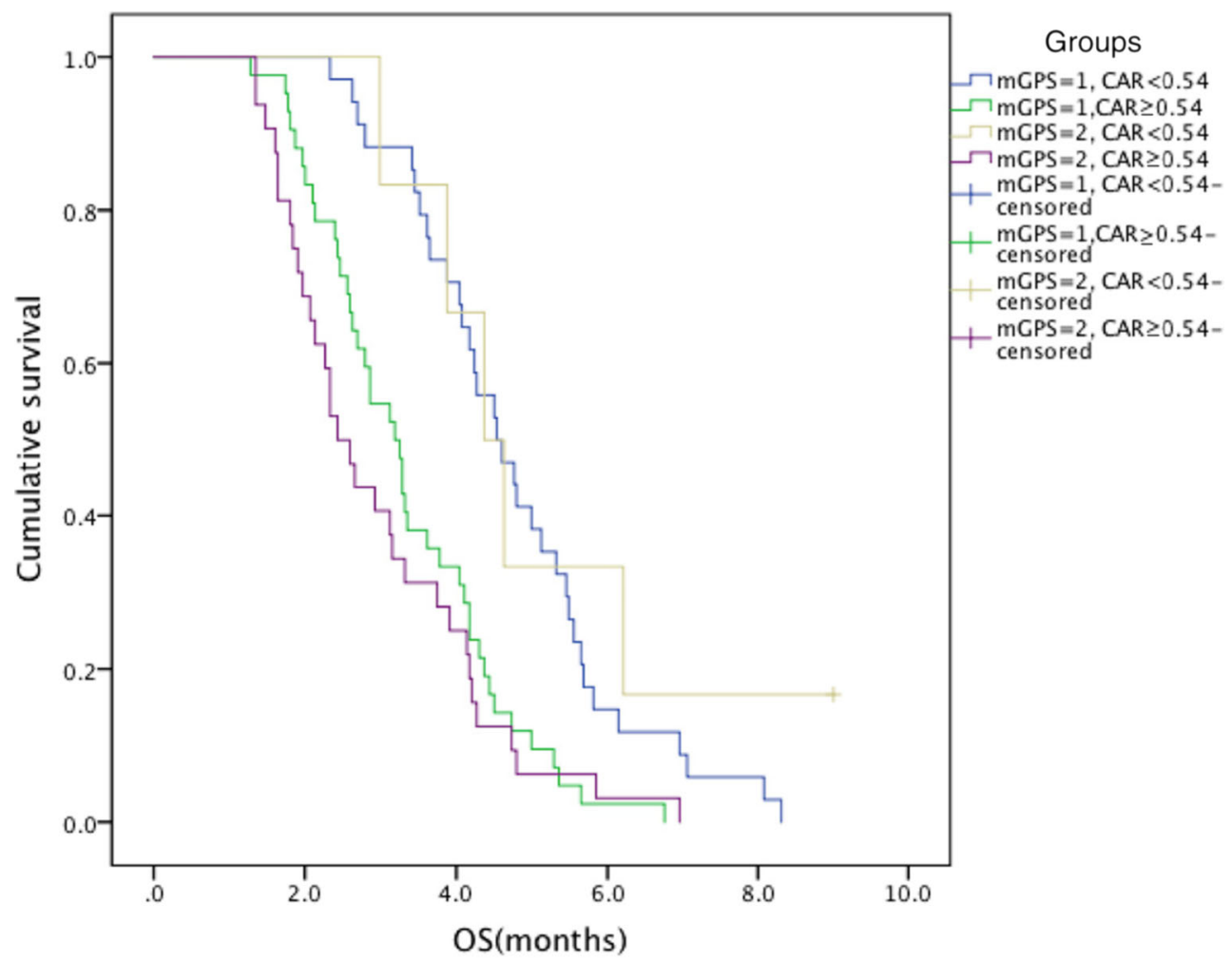

Fig. 4 Kaplan-Meier curves between groups based on the mGPS scores and CRP/Alb ratio 
comprehensive evaluation of these inflammatory parameters could provide a more advisable prognostic estimate. In accordance with the result of the chi-square test of the $\mathrm{CRP} / \mathrm{Alb}$ ratio versus disease stage, the $\mathrm{CRP} / \mathrm{Alb}$ ratio remained a significant prognostic parameter regardless of the stage of advanced PC in subgroup analyses. After excluding the confounding factors from the multivariate analyses, the CRP/Alb ratio remained the only significant inflammation-related prognostic index. To the best of our knowledge, this is the first study to explore the role of the $\mathrm{CRP} / \mathrm{Alb}$ ratio as a predictor of prognosis in advanced PC.

As the mGPS and CRP/Alb ratio both include the CRP and albumin levels, comparison of the two factors was performed. Because the $\mathrm{CRP} / \mathrm{Alb}$ ratios of patients with mGPS scores of 0 were all less than 0.54 , dichotomizing patients with mGPS scores of 1 and 2 on the basis of the $\mathrm{CRP} / \mathrm{Alb}$ ratio was conducted. Kaplan-Meier tests showed that patients with an mGPS score of 1 and CRP/ Alb ratio $<0.54$ and an mGPS score of 2 and CRP/Alb ratio $<0.54$ had comparable longer OS. Similarly, those in the groups with an mGPS score of 1 and CRP/Alb ratio $\geq 0.54$ and an mGPS score of 2 and CRP/Alb ratio $\geq 0.54$ had parallel poor outcome. The results exposed defects in the mGPS prognostic ability for patients with scores of 1 and 2 because they did not have significant differences in OS; by contrast, they could be distinguished according to the CRP/Alb ratio. This disadvantage arises from its nature as a categorized variable that fails to accurately reflect the disease condition of every patient. In conclusion, the $\mathrm{CRP} / \mathrm{Alb}$ ratio is superior to the other inflammation-related prognostic factors. Our findings may have practical value in the therapy of advanced PC patients. Patients with a high CRP/Alb ratio may require more active adjuvant chemotherapy.

The generalizability of the conclusions is limited by the threatened independence of the variables. As a retrospective and single-center study, the limitations of the current research lie in its intrinsic features. To narrow down the inevitable selection bias, we enrolled consecutive patients and included a relatively large sample size. Meanwhile, we explored the prognostic significance of the CRP/Alb ratio in a multifaceted approach, including validation of the value at the level of all patients and patient subgroups based on disease stage. However, a multicenter prospective validation study with a larger scale sample is needed to confirm our findings.

\section{Compliance with ethical standards}

Ethics The study protocol was approved by the ethics committee of Southern Medical University, and written informed consent for the utilization of clinical data was obtained from all enrolled patients.

Conflicts of interest None
Open Access This article is distributed under the terms of the Creative Commons Attribution 4.0 International License (http:// creativecommons.org/licenses/by/4.0/), which permits unrestricted use, distribution, and reproduction in any medium, provided you give appropriate credit to the original author(s) and the source, provide a link to the Creative Commons license, and indicate if changes were made.

\section{References}

1. Torre LA, Bray F, Siegel RL, Ferlay J, Lortet-Tieulent J, Jemal A. Global cancer statistics, 2012. CA Cancer J Clin. 2015;65:87-108.

2. WHO | Mortality. WHO [Internet]. World Health Organization; 2014. Available from: http:/www.who.int/topics/mortality/en/

3. Grivennikov SI, Greten FR, Karin M. Immunity, inflammation, and cancer. Cell. 2010;140:883-99.

4. Allavena P, Garlanda C, Borrello MG, Sica A, Mantovani A. Pathways connecting inflammation and cancer. Curr Opin Genet Dev. 2008;18:3-10.

5. Mantovani A, Allavena P, Sica A, Balkwill F. Cancer-related inflammation. Nature. 2008;454:436-44.

6. Shalapour S, Karin M. Immunity, inflammation, and cancer: an eternal fight between good and evil. J Clin Invest. 2015;125:3347-55.

7. Stotz M, Gerger A, Eisner F, Szkandera J, Loibner H, Ress AL, et al. Increased neutrophil-lymphocyte ratio is a poor prognostic factor in patients with primary operable and inoperable pancreatic cancer. Br J Cancer. 2013;109:416-21.

8. Zhou T, Zhan J, Hong S, Hu Z, Fang W, Qin T, et al. Ratio of Creactive protein/albumin is an inflammatory prognostic score for predicting overall survival of patients with small-cell lung cancer. Nat Publ Group. 2015;5:10481.

9. Gomez D, Farid S, Malik HZ, Young AL, Toogood GJ, Lodge JPA, et al. Preoperative neutrophil-to-lymphocyte ratio as a prognostic predictor after curative resection for hepatocellular carcinoma. World J Surg. 2008;32:1757-62.

10. Ni X-C, Yi Y, Fu Y-P, He H-W, Cai X-Y, Wang J-X, et al. Prognostic value of the modified Glasgow prognostic score in patients undergoing radical surgery for hepatocellular carcinoma. Medicine (Baltimore). 2015;94:e1486.

11. Hong S, Zhou T, Fang W, Xue C, Hu Z, Qin T, et al. The prognostic nutritional index (PNI) predicts overall survival of small-cell lung cancer patients. Tumour Biol. 2015;36:3389-97.

12. Nakamura K, Nishida T, Haruma T, Haraga J, Omichi C, Ogawa C, et al. Pretreatment platelet-lymphocyte ratio is an independent predictor of cervical cancer recurrence following concurrent chemoradiation therapy. Mol Clin Oncol. 2015;3:1001-6.

13. Kinoshita A, Onoda H, Imai N, Iwaku A, Oishi M, Tanaka K, et al. The C-reactive protein/albumin ratio, a novel inflammation-based prognostic score, predicts outcomes in patients with hepatocellular carcinoma. Ann Surg Oncol. 2015;22:803-10.

14. Xu X-L, Yu H-Q, Hu W, Song Q, Mao W-M. A novel inflammation-based prognostic score, the C-reactive protein/ albumin ratio predicts the prognosis of patients with operable esophageal squamous cell carcinoma. PLoS One. 2015;10: e0138657.

15. Wei X-L, Wang F-H, Zhang D-S, Qiu M-Z, Ren C, Jin Y, et al. A novel inflammation-based prognostic score in esophageal squamous cell carcinoma: the C-reactive protein/albumin ratio. BMC Cancer. 2015;15:350.

16. Liu X, Sun X, Liu J, Kong P, Chen S, Zhan Y, et al. Preoperative Creactive protein/albumin ratio predicts prognosis of patients after curative resection for gastric cancer. Transl Oncol. 2015;8:339-45. 
17. Fairclough E, Cairns E, Hamilton J, Kelly C. Evaluation of a modified early warning system for acute medical admissions and comparison with C-reactive protein/albumin ratio as a predictor of patient outcome. Clin Med (Lond). 2009;9:30-3.

18. Proctor MJ, Morrison DS, Talwar D, Balmer SM, O'Reilly DSJ, Foulis AK, et al. An inflammation-based prognostic score (mGPS) predicts cancer survival independent of tumour site: a Glasgow Inflammation Outcome Study. Br J Cancer. 2011;104:726-34.

19. Wang D-S, Luo H-Y, Qiu M-Z, Wang Z-Q, Zhang D-S, Wang F-H, et al. Comparison of the prognostic values of various inflammation based factors in patients with pancreatic cancer. Med Oncol. 2012;29:3092-100.

20. Garcea G, Ladwa N, Neal CP, Metcalfe MS, Dennison AR, Berry DP. Preoperative neutrophil-to-lymphocyte ratio (NLR) is associated with reduced disease-free survival following curative resection of pancreatic adenocarcinoma. World J Surg. 2011;35:868-72.

21. Budczies J, Klauschen F, Sinn BV, Gyorffy B, Schmitt WD, DarbEsfahani S, et al. Cutoff Finder: a comprehensive and straightforward Web application enabling rapid biomarker cutoff optimization. PLoS One. 2012;7:e51862.

22. Balkwill F, Mantovani A. Inflammation and cancer: back to Virchow? Lancet. 2001;357:539-45.

23. Elinav E, Nowarski R, Thaiss CA, Hu B, Jin C, Flavell RA. Inflammation-induced cancer: crosstalk between tumours, immune cells and microorganisms. Nat Rev Cancer. 2013;13:759-71.

24. McKeown DJ, Brown DJF, Kelly A, Wallace AM, McMillan DC. The relationship between circulating concentrations of Creactive protein, inflammatory cytokines and cytokine receptors in patients with non-small-cell lung cancer. Br J Cancer. 2004;91:1993-5.

25. Miura T, Mitsunaga S, Ikeda M, Shimizu S, Ohno I, Takahashi H, et al. Characterization of patients with advanced pancreatic cancer and high serum interleukin-6 levels. Pancreas. 2015;44:756-63.

26. Szkandera J, Stotz M, Absenger G, Stojakovic T, Samonigg H, Kornprat P, et al. Validation of C-reactive protein levels as a prognostic indicator for survival in a large cohort of pancreatic cancer patients. Br J Cancer. 2014;110:183-8.

27. Pine JK, Fusai KG, Young R, Sharma D, Davidson BR, Menon KV, et al. Serum C-reactive protein concentration and the prognosis of ductal adenocarcinoma of the head of pancreas. Eur J Surg Oncol. 2009;35:605-10.

28. Kishi T, Nakamura A, Itasaka S, Shibuya K, Matsumoto S, Kanai $\mathrm{M}$, et al. Pretreatment C-reactive protein level predicts outcome and patterns of failure after chemoradiotherapy for locally advanced pancreatic cancer. Pancreatology. 2015;15:694-700.

29. Kowalski-Saunders PW, Winwood PJ, Arthur MJ, Wright R. Reversible inhibition of albumin production by rat hepatocytes maintained on a laminin-rich gel (Engelbreth-Holm-Swarm) in response to secretory products of Kupffer cells and cytokines. Hepatology. 1992;16:733-41.

30. Barber MD, Ross JA, Fearon KC. Changes in nutritional, functional, and inflammatory markers in advanced pancreatic cancer. Nutr Cancer. 1999;35:106-10.

31. Fanali G, di Masi A, Trezza V, Marino M, Fasano M, Ascenzi P. Human serum albumin: from bench to bedside. Mol Asp Med. 2012;33:209-90.

32. Nazha B, Moussaly E, Zaarour M, Weerasinghe C, Azab B. Hypoalbuminemia in colorectal cancer prognosis: nutritional marker or inflammatory surrogate? World J Gastrointest Surg. 2015;7:370-7.

33. Boonpipattanapong T, Chewatanakornkul S. Preoperative carcinoembryonic antigen and albumin in predicting survival in patients with colon and rectal carcinomas. J Clin Gastroenterol. 2006;40:592-5.

34. Siddiqui A, Heinzerling J, Livingston EH, Huerta S. Predictors of early mortality in veteran patients with pancreatic cancer. Am J Surg. 2007;194:362-6.

35. Onate-Ocana LF, Aiello-Crocifoglio V, Gallardo-Rincon D, Herrera-Goepfert R, Brom-Valladares R, Carrillo JF, et al. Serum albumin as a significant prognostic factor for patients with gastric carcinoma. Ann Surg Oncol. 2007;14:381-9. 